\title{
A light Higgs boson would invite supersymmetry
}

\author{
John Ellis, Douglas Ross ${ }^{1}$ \\ TH Division, CERN, Geneva, Switzerland
}

Received 13 December 2000; accepted 18 January 2001

Editor: R. Gatto

\begin{abstract}
If the Higgs boson weighs about $115 \mathrm{GeV}$, the effective potential of the Standard Model becomes unstable above a scale of about $10^{6} \mathrm{GeV}$. This instability may be rectified only by new bosonic particles such as stop squarks. However, avoiding the instability requires fine-tuning of the model couplings, in particular if the theory is not to become non-perturbative before the Planck scale. Such fine-tuning is automatic in a supersymmetric model, but is lost if there are no higgsinos. A light Higgs boson would be prima facie evidence for supersymmetry in the top-quark and Higgs sectors. ( ) 2001 Elsevier Science B.V. All rights reserved.
\end{abstract}

\section{Introduction}

The LEP Collaborations [1] and the LEP Working Group for Higgs boson searches [2] have recently reported excesses of events that might be due to the production of a Higgs boson weighing about $115 \mathrm{GeV}$. Confirmation of this exciting interpretation of their events may have to wait for several years, but we already find the hint sufficiently encouraging to explore the possible implications of such a discovery. As has already been pointed out [3], the existence of such a light Higgs boson would imply that the Standard Model could remain valid only up to scales limited by about $10^{6} \mathrm{GeV}$ [4]. This is because the effective Higgs potential would be destabilised by the radiative corrections due to the relatively heavy top quark, that could not be counterbalanced by those due to a relatively light Higgs boson alone,

\footnotetext{
E-mail address: john.ellis@cern.ch (J. Ellis).

1 On leave of absence from: Department of Physics and Astronomy, University of Southampton, Southampton SO17 1BJ, UK.
}

necessitating the appearance of new physics. Extant non-perturbative models of new physics, such as technicolour, cannot accommodate a relatively light Higgs boson [5]. On the other hand, a relatively light Higgs boson favours prima facie generically a perturbative scenario for electroweak symmetry breaking. Among these, it is well known that a Higgs boson weighing less than about $130 \mathrm{GeV}$ is expected in the minimal supersymmetric extension of the Standard Model (MSSM) [6].

The question we address in this paper is the extent to which a Higgs boson weighing about $115 \mathrm{GeV}$ would actually require significant features of supersymmetry. Salient features of the MSSM that one might look for include (a) bosonic partners for known fermions, and vice versa, (b) the absence of certain renormalization effects, entailed in the MSSM by relations between bosonic and fermionic couplings, and (c) soft supersymmetry breaking at a scale of about $10^{3} \mathrm{GeV}$.

Remarkably, we find that a $115 \mathrm{GeV}$ Higgs boson would provide non-trivial hints for all these characteristics of the MSSM. Specifically, analyzing the renormalization of the effective potential, we find that 
(a) the new physics that must appear at some energy scale below $10^{6} \mathrm{GeV}$ has to contain a dominant bosonic component, just as provided by the stop squarks in the MSSM, (b) that this new component must be finely tuned, just as occurs when supersymmetry relates the couplings of bosons and fermions in the top and Higgs supermultiplets, (c) that the splitting of bosonic and fermionic partners should be in the range $10^{2}$ to $10^{5} \mathrm{GeV}$. Further, analyzing precision electroweak observables, we show (d) that the effective mass splitting between members of a scalar isomultiplet must be small, as happens in the MSSM. The new physics may not be supersymmetric, but it must share many features with supersymmetry.

\section{Renormalization of the effective potential}

We first consider in more detail the renormalization of the effective Higgs potential. In the minimal Standard Model, there are important renormalization effects of the quartic Higgs self-coupling $\lambda_{H}$ due to the top-quark Yukawa coupling $g_{t}$, as well as those due to $\lambda_{H}$ itself [8]:

$$
\begin{aligned}
& \beta_{\lambda_{H}} \equiv \frac{\partial}{\partial \mu} \lambda_{H} \\
& =\frac{1}{16 \pi^{2}}\left(4 \lambda_{H}^{2}-36 g_{t}^{4}+\frac{27}{4} g^{4}\right. \\
& \left.+\frac{9}{4} g^{\prime 4}+\frac{9}{2} g^{2} g^{\prime 2}\right)+2 \lambda_{H} \gamma_{H},
\end{aligned}
$$

where $\gamma_{H}$ is the one-loop wave-function renormalization constant of the Higgs field:

$\gamma_{H}=\frac{1}{16 \pi^{2}}\left(6 g_{t}^{2}-\frac{9}{2} g^{2}-\frac{3}{2} g^{\prime 2}\right)$,

where $g$ and $g^{\prime}$ are the $S U(2)$ and $U(1)$ gauge couplings, respectively. We neglect all the other Yukawa couplings.

The effective potential of the Standard Model becomes unstable at a value of the Higgs field $H$ very close to that where $\lambda_{H}$ turns negative. With $g_{t}$ fixed so that $m_{t}=175 \mathrm{GeV}$ and $\lambda_{H}$ fixed so that $m_{H}=115 \mathrm{GeV}$, this instability scale is no more than $10^{6} \mathrm{GeV}$, because the relatively large value of $g_{t}$, which tries to decrease $\lambda_{H}$, overwhelms the relatively small value of $\lambda_{H}$ itself. Thus, as already mentioned, new physics is needed to stabilise our electroweak vacuum.

What form might this new physics take? Looking at the signs in (1), it is clear that adding new fermions, e.g., in a fourth generation, would only exacerbate the situation. Only new bosonic physics can fit the bill. The symmetry properties of this new physics are not highly constrained, a priori, and one could imagine introducing combinations of $N_{I, Y}$ new bosonic multiplets of isospin $I$ and weak hypercharge $Y$. We recall that the MSSM contains an $N_{1 / 2,-1 / 6}=N_{C}=3$ colour-triplet isospin doublets of left-handed stop and sbottom squarks $\left(\tilde{t}_{L}, \tilde{b}_{L}\right)$ and $N_{0,2 / 3}=N_{0,-1 / 3}=$ $N_{C}=3$ isospin singlets $\tilde{t}_{R}$ and $\tilde{b}_{R}$, but we shall not postulate such a combination at the outset. Moreover, in the MSSM all the quartic couplings are strictly related to $g_{t}$ and the gauge couplings, but we shall also not postulate such relations a priori.

To begin with, we consider the most general addition of $n$ (complex) multiplets of scalar particles which each carry the same weak isospin $I$ and weak hypercharge $Y$. These scalar particles are assumed not to partake in the symmetry-breaking mechanism and so do not possess v.e.v.'s. Denoting these scalars by $\phi_{i}^{\alpha}$, where $i$ is the third component of isospin and $\alpha$ runs over the number of copies, this gives rise to extra terms in the Lagrangian

$$
\begin{aligned}
\mathcal{L}^{\phi}= & \left|D_{\mu} \phi^{\alpha}\right|^{2}-\left(M^{2}-M_{0}^{2}\right)\left|\phi^{\alpha}\right|^{2} \\
& -\frac{2 M_{0}^{2}}{v^{2}}|H|^{2}\left|\phi^{\alpha}\right|^{2} \\
& -\frac{4\left(\Delta M^{2}\right)}{v^{2}}\left(H^{\dagger} \tau^{a} H\right)\left(\phi^{\dagger \alpha} T^{a} \phi^{\alpha}\right) \\
& -\frac{\lambda_{\phi}}{6}\left(\left|\phi^{\alpha}\right|^{2}\right)^{2},
\end{aligned}
$$

where the $\tau^{a}$ are the defining representation of the $S U(2)$ generators, the $T^{a}$ are the isospin $I$ representation and $v$ is the v.e.v. of the Higgs field. We see in (3) that there are three contributions to the masses of the added scalar particles - a conventional quadratic term and two contributions from couplings to the Higgs field. The quadratic term, $M$, sets the overall scale of the new bosonic physics, and generalizes the soft supersymmetry-breaking terms postulated in the MSSM. One of the Higgs quartic contributions in (3) conserves custodial $S U(2)$ and contributes an average mass $M_{0}$ to the multiplet, and the other breaks $S U(2)$, 
splitting the squared masses of members of the multiplet with adjacent values of $I_{3}$ by $\left(\Delta M^{2}\right)$. This term may have either sign.

\section{Stability of the effective potential}

The added scalar particles make a contribution to the one-loop $\beta$ function for $\lambda_{H}$ (above the threshold $\mu=M)$ of the form

$$
\begin{aligned}
\Delta \beta_{\lambda_{H}}=\frac{6 n}{4 \pi^{2} v^{4}} & ( \\
& (2 I+1) M_{0}^{4} \\
& \left.+\left(\Delta M^{2}\right)^{2} \frac{I(I+1)(2 I+1)}{3}\right) .
\end{aligned}
$$

Before one can insert this into the R.G.E. equation for $\lambda_{H}$, one needs the $\beta$ functions for $M_{0}^{2}$ and $\left(\Delta M^{2}\right)$, which, at the one-loop level, are given by:

$$
\begin{array}{r}
\beta_{M_{0}^{2}}=\frac{M_{0}^{2}}{16 \pi^{2}}\left\{2 \lambda_{H}+\frac{4 n}{3}(I+1) \lambda_{\phi}+8 \frac{M_{0}^{2}}{v^{2}}\right. \\
\left.+\frac{3}{2}\left(g^{4} I(I+1)+g^{\prime 4} Y^{2}\right)\right\} \\
+M_{0}^{2}\left(\gamma_{H}+\gamma_{\phi}\right), \\
\beta_{\left(\Delta M_{0}^{2}\right)}=\frac{\left(\Delta M^{2}\right)}{16 \pi^{2}}\left\{2 \lambda_{H}+\frac{4 n}{3}(I+1) \lambda_{\phi}\right. \\
\left.+8 \frac{M_{0}^{2}}{v^{2}}-3 Y g^{2} g^{\prime 2}\right\} \\
+\left(\Delta M^{2}\right)\left(\gamma_{H}+\gamma_{\phi}\right),
\end{array}
$$

where $\gamma_{\phi}$ is the one-loop wave-function renormalization constant of the scalar field,

$\gamma_{\phi}=\frac{1}{16 \pi^{2}}\left(-6 I(I+1) g^{2}-6 Y^{2} g^{\prime 2}\right)$.

Finally, the one-loop $\beta$ function for the quartic selfcoupling $\lambda_{\phi}$ is

$$
\begin{aligned}
\beta_{\lambda_{\phi}}= & \frac{1}{16 \pi^{2}}\left\{48 \frac{M_{0}^{4}}{v^{4}}+\frac{2}{3} \lambda_{\phi}^{2}((2 I+1) n+4)\right. \\
& +3 g^{4} I(I+1)\left(4 I^{2}+6 I-1\right) \\
& \left.+36 g^{\prime 4} Y^{4}+9 g^{2} g^{\prime 2}(2 I+1) Y^{2}\right\} \\
& +2 \lambda_{\phi} \gamma_{\phi} .
\end{aligned}
$$

The resulting set of coupled differential equations can readily be solved by numerical methods.
We note that, as required, the extra contributions to the renormalization of the Higgs quartic coupling are positive, thereby counteracting the large contribution in (1) from the large top-quark Yukawa coupling $g_{t}$. However, the effect of these extra terms is sensitive to the quartic self-coupling, $\lambda_{\phi}$, since this drives the running of $M_{0}^{2}$ and $\left(\Delta M^{2}\right)$, which in turn drive the running of $\lambda_{H}$. It would be unnatural for such a coupling to be too small.

As an example, we have taken the case of six isodoublets with $Y=1 / 2$, and set $\lambda_{\phi}=\lambda_{H}$ at $M_{Z} \cdot{ }^{2}$ We can see from Fig. 1 that, as one increases the coupling between $\phi$ and $H$, and hence the contribution $M_{0}$ to the scalar masses from the coupling with the Higgs field, the critical scale $\mu_{c}$ at which the Higgs coupling changes sign increases from its starting value of (just below) $10^{6} \mathrm{GeV}$, which is attained for small $M_{0}$. However, one can see quite dramatically in Fig. 2 what happens when we try to increase the value of $M_{0}$ so that this instability of the effective potential does not occur below the Planck scale. The fine-tuning required is so acute that, as one increases $M_{0}$ from $70.9 \mathrm{GeV}$ to $71.0 \mathrm{GeV}$, the behaviour of $\lambda_{H}(\mu)$ as a function of $\mu$ switches from one that becomes negative at $\mu \sim 5 \times 10^{7} \mathrm{GeV}$ to one that reverses its fall just below that scale, and becomes so large at the Planck scale that perturbative calculations become unreliable.

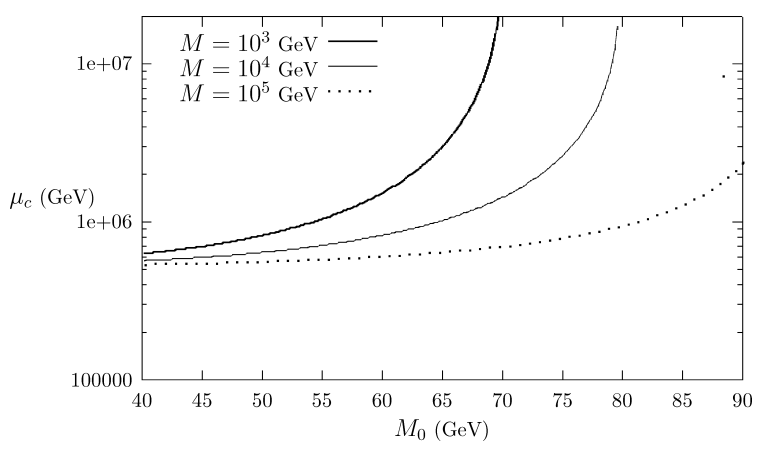

Fig. 1. Examples of the increase in the critical scale $\mu_{c}$ at which the effective potential becomes unstable, as the coupling $M_{0}$ between the added scalar field $\phi$ and the Higgs field $H$ is increased, for three different choices of the scale $M$ of new physics.

2 This choice is motivated by the MSSM with its $\left(\tilde{t}_{L}, \tilde{b}_{L}\right), \tilde{t}_{R}$ and $\tilde{b}_{R}$. We have checked that similar conclusions hold if we use just three 'coloured' isodoublets. The main features of Figs. 1 and 2 are somewhat accentuated, whereas Fig. 3 is essentially unchanged. 


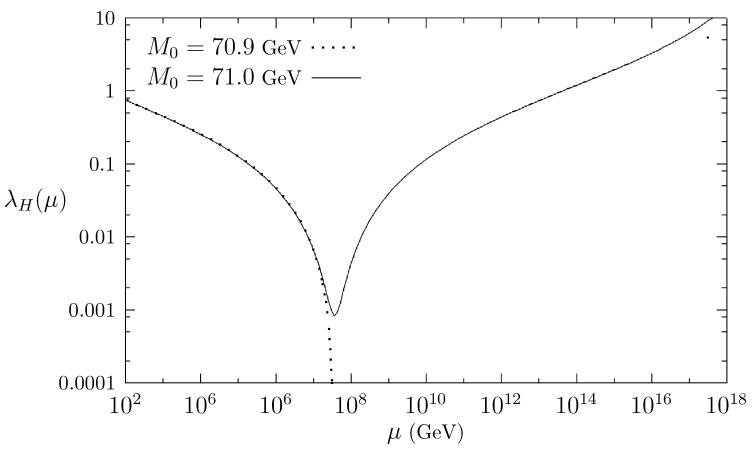

Fig. 2. An example of the acute fine-tuning of $M_{0}$ that arises when one attempts to couple the added scalars to the Higgs fields in such a way that the effective potential remains stable, whilst at the same time remaining within the perturbative regime. Here the new physics scale $M$ is taken to be $1 \mathrm{TeV}$.

The absence of new bosonic particles at LEP and the Tevatron indicates that $M \gtrsim 100 \mathrm{GeV}$. On the other hand, it is clear that the value of $M_{0}$ required to stabilize the effective potential must increase as $M$ increases. However, $M$ must be less than $10^{6} \mathrm{GeV}$, since this is the scale at which the instability appears in the Standard Model, and $M_{0}$ is limited by the electroweak scale. Since one would expect $M_{0}$ to be determined by the weak-interaction scale, it is apparent from Fig. 1 that the scale $M$ at which new physics is introduced could not be much larger than $10^{4} \mathrm{GeV}$, and the fine-tuning visible in Fig. 2 is exacerbated for larger $M$. For this reason, we would argue that $M \lesssim 10^{5} \mathrm{GeV}$.

\section{Fine-tuning}

Supersymmetry is the only known theory that provides the requisite fine-tuning in a natural way. In a supersymmetric theory, the quartic Higgs coupling is forced by supersymmetry to take the value

$\lambda_{H}=\frac{3}{4}\left(g^{2}+g^{\prime 2}\right)$

at the tree level, and its running is determined by the $\beta$ functions of the $S U(2)$ and $U(1)$ gauge couplings.

We can attempt to mimic such a theory by starting from the Standard Model with a Higgs mass of $115 \mathrm{GeV}$, and using the R.G.E. of the Standard Model to run the effective potential up to $1.4 \mathrm{TeV}$, which is the scale where the Standard Model $\lambda_{H}$ has the value specified by the relation (9). It is at this point that we introduce six new isodoublets of scalar particles that mimic the third generation of (left- and right-handed) squarks. In order for the quartic Higgs coupling to run as the required combination of gauge couplings, we need to impose the following relation on $M_{0}:{ }^{3}$

$$
\begin{aligned}
M_{0}^{4}=\frac{v^{4}}{8}\{ & \left(g_{t}^{2}-\frac{\left(g^{2}+g^{\prime 2}\right)}{8}\right)^{2} \\
& \left.+\left(\frac{135 g^{4}+231 g^{\prime 4}-18 g^{2} g^{\prime 2}}{576}\right)\right\} .
\end{aligned}
$$

The important feature of this relation is the fact that, when substituted into (4), the terms proportional to the top-quark Yukawa coupling cancel those of (1). In order for this relation to be maintained as the couplings run, we need to impose the condition

$\lambda_{\phi}=\frac{g_{t}^{2}}{12}-\frac{4}{9} g_{3}^{2}$,

where $g_{3}$ is the QCD coupling, and we have dropped some terms proportional to powers of $g$ and $g^{\prime}$, which have a negligible effect.

In contrast, in a supersymmetric model, the couplings are guaranteed to run in the required fixed ratio, because of contributions to the $\beta$ functions from loops involving higgsino and gaugino particles. However, in our mimicry, such extra fermions have not been added. The running of the quartic Higgs coupling in a supersymmetric model receives a contribution

$$
\frac{1}{16 \pi^{2}}\left\{-3\left(5 g^{4}+2 g^{2} g^{\prime 2}+g^{\prime 4}\right)+2 \lambda_{H}\left(3 g^{2}+g^{\prime 2}\right)\right\}
$$

from these fermionic superpartners, whereas the supersymmetric one-loop $\beta$ function for the top-quark Yukawa coupling [9] has a contribution

$\frac{3}{16 \pi^{2}} g_{t}^{3}$

from a loop involving a higgsino and

$\frac{8}{48 \pi^{2}} g_{t} g_{3}^{2}$

\footnotetext{
3 This awkward expression arises because, in a genuine supersymmetric model, the left- and right-handed squarks couple to the Higgs field in different ways, as reflected in the correct matter parts of the $\beta$ functions for the gauge couplings. In our mimicry, we have assumed for simplicity that all the scalar particles couple in the same way.
} 


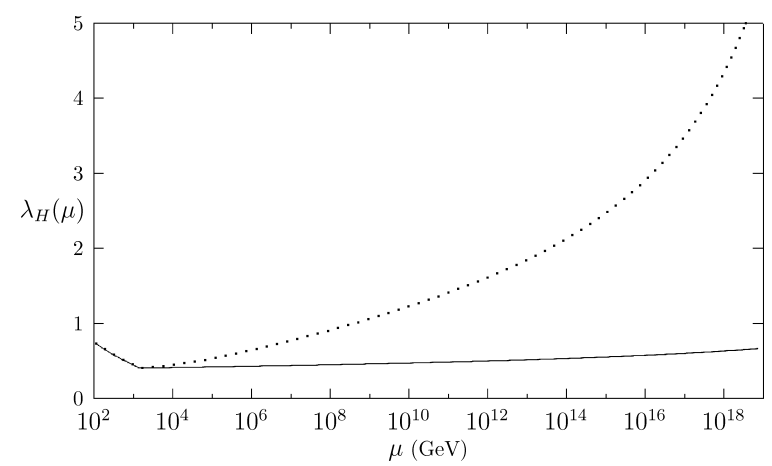

Fig. 3. An example of the role played by fermionic superpartners in the running of $\lambda_{H}$. The solid line corresponds to a genuine supersymmetric model, whereas the dotted line gives the running of the quartic Higgs coupling when the contributions from fermionic higgsino and gaugino superpartners have been removed.

from a loop involving a gluino. Moreover, the $\beta$ functions for the gauge couplings [10] also receive contributions from these fermionic superpartners.

The essential role played by these fermionic superpartners can be seen in Fig. 3, in which we compare the running of $\lambda_{H}$ in the case of a genuine supersymmetric model with that in our mimic model, that contains the required number of extra scalar multiplets with couplings tuned at the erstwhile supersymetric threshold to coincide with the relations forced by supersymmetry. In the genuine case, there is a very small rise in the quartic coupling above the supersymmetric threshold, due to the fact that the $\beta$ functions for $g$ and $g^{\prime}$ have positive values. On the other hand, we can see quite clearly that, when we remove the contributions from the fermionic superpartners, we are again faced with the problem of a quartic coupling that rises too fast.

We note that most of this effect is due to diagrams involving an internal higgsino, whereas those involving gauginos have only a small effect on the running of $\lambda_{H}$.

\section{Electroweak radiative corrections}

A further constraint on the parameters involving additional scalar multiplets arises from consideration of the precision weak parameters [7] $S$ and $T$, that characterize 'oblique' weak corrections.

We recall that scalar isospin multiplets with exact mass degeneracy, do not make any contribution to these parameters. However, the $S U(2)$-breaking mass term $\propto\left(\Delta M^{2}\right)$ discussed above does introduce contributions to $S$ and $T$, which are given by

$$
\begin{aligned}
\Delta S= & \frac{n Y}{\pi} \sum_{i=-I}^{I} i \ln (1+\eta i), \\
\Delta T= & \frac{n\left(\Delta M^{2}\right)}{8 \pi M_{W}^{2} \sin ^{2} \theta_{W}} \sum_{i=-I}^{I}(I(I+1)-i(i-1)) \\
& \times\{\eta \ln (1+i \eta)+1+\eta(i-1 / 2) \\
& \left.\quad-\frac{(1+\eta(i-1))^{2}}{\eta} \ln \left(\frac{1+\eta i}{1+\eta(i-1)}\right)\right\},
\end{aligned}
$$

where $\eta=\left(\Delta M^{2}\right) / M^{2}$ is the fractional mass-splitting between members of the multiplet. These expressions simplify to

$$
\begin{aligned}
& \Delta S=\frac{n Y}{9 \pi} \frac{\left(\Delta M^{2}\right)}{M^{2}} I(I+1)(2 I+1), \\
& \Delta T=\frac{n Y}{6 \pi \sin ^{2} \theta_{W}} \frac{\left(\Delta M^{2}\right)}{M_{W}^{2}} I(I+1)(2 I+1),
\end{aligned}
$$

in the limit $\eta \ll 1$.

The most stringent limit from precision electroweak data is that on the quantity $T$. From the experimental error on this quantity we conclude that any new physics can contribute at most $\pm 0.14 .{ }^{4}$ This means, for example, that if one were to add six isodoublets, the quantity $\left(\Delta M^{2}\right)$ could be no greater than $400 \mathrm{GeV}^{2}$. This implies, in terms of quartic couplings, that the ratio of the $S U$ (2)-breaking quartic coupling to the Higgs quartic coupling $\lambda_{H}$ could be no more than $0.04{ }^{5}$ Such an unnatural fine-tuning would not normally survive the higher-order corrections due to $\lambda_{H}$.

However, supersymmetry provides a natural suppression of this effect. Provided that the soft supersymmetry-breaking mass is large compared with the contribution to the squark masses due to the Yukawa couplings, the squark mass eigenstates have $S U(2)$ mass

\footnotetext{
${ }^{4}$ Note that, since $\left(\Delta M^{2}\right)$ can take either sign, the contribution to $T$ can also take either sign. Thus, one could arrange a conspiracy to produce cancellations with further new physics at some much higher scale, but here we neglect such a possibility.

5 Alternatively and equivalently, if $M_{0} \sim m_{t} \sim 200 \mathrm{GeV}$, one must enforce $\left(\Delta M^{2}\right) / M_{0}^{2} \lesssim 0.01$.
} 
splittings which although considerably larger than the above-mentioned limit, are almost equal and opposite, so that the contribution to $T$ is comfortably small.

\section{Conclusions}

We have learnt in this Letter that, if $m_{H}=115 \mathrm{GeV}$ and we want the effective Higgs potential to remain stable all the way up to the Planck scale of $10^{19} \mathrm{GeV}$, the Standard Model must be supplemented by a remarkably supersymmetric-seeming set of new physics. The top quark must be accompanied by one or more scalar multiplets, whose couplings must be tuned very finely if the effective potential is to steer a course between the Scylla of a negative scalar coupling and the Charybdis of non-perturbative couplings. This fine-tuning is provided in a supersymmetric model by the stop and higgsino supersymmetric partners of the top quark and the Higgs boson, and the characteristic relations between couplings that they preserve. Thus, a light Higgs could be construed as prima facie evidence for the supersymmetrization of the top quark and the Higgs boson.

In order to extend the sell-by scale of the Standard Model far beyond $10^{6} \mathrm{GeV}$, the parameters of the new physics must be quite finely tuned. Supersymmetry is one example of new physics that fulfills all the requirements: any alternative should quack in a similar way.

\section{Acknowledgement}

We thank Ben Allanach for helpful discussions.

\section{References}

[1] ALEPH Collaboration, http://alephwww.cern.ch/ and preprint CERN-EP/2000-138, hep-ex/0011045;

DELPHI Collaboration, http://delphi.web.cern.ch/Delphi/;

L3 Collaboration, http://13www.cern.ch/ and preprint CERNEP/2000-140, hep-ex/0011043;

OPAL Collaboration, http://opal.web.cern.ch/Opal/ PPwelcome.html, and Physics Note PN468, http://opal. web.cern.ch/Opal/pubs/physnote/html/pn468.html.

[2] P. Igo-Kemenes, for the LEP Working Group on Higgs boson searches, Talk at the LEPC open session 3 November, 2000, http://lephiggs.web.cern.ch/LEPHIGGS/talks/index.html.

[3] J. Ellis, G. Ganis, D.V. Nanopoulos, K.A. Olive, hep-ph/ 0009355;

See also G.L. Kane, S.F. King, L.-T. Wang, hep-ph/0010312.

[4] N. Cabibbo, L. Maiani, G. Parisi, R. Petronzio, Nucl. Phys. B 158 (1979) 295;

M. Lindner, Z. Phys. C 31 (1986) 295;

M. Lindner, M. Sher, H.W. Zaglauer, Phys. Lett. B 228 (1989) 139;

G. Altarelli, G. Isidori, Phys. Lett. B 337 (1994) 141;

J.A. Casas, J.R. Espinosa, M. Quirós, Phys. Lett. B 342 (1995) 171

J.A. Casas, J.R. Espinosa, M. Quirós, Phys. Lett. B 382 (1996) 374.

[5] E. Farhi, L. Susskind, Phys. Rep. 74 (1981) 277.

[6] J. Ellis, G. Ridolfi, F. Zwirner, Phys. Lett. B 257 (1991) 83; M.S. Berger, Phys. Rev. D 41 (1990) 225;

Y. Okada, M. Yamaguchi, T. Yanagida, Prog. Theor. Phys. 85 (1991) 1 ;

Y. Okada, M. Yamaguchi, T. Yanagida, Phys. Lett. B 262 (1991) 54;

H.E. Haber, R. Hempfling, Phys. Rev. Lett. 66 (1991) 1815.

[7] M.E. Peskin, T. Takeuchi, Phys. Rev. D 46 (1992) 381; G. Altarelli, R. Barbieri, S. Jadach, Nucl. Phys. B 369 (1992) 3.

[8] C. Ford, D.R.T. Jones, P.W. Stephenson, M.B. Einhorn, Nucl. Phys. B 395 (1993) 17.

[9] S.P. Martin, M.T. Vaughn, Phys. Rev. D 50 (1994) 2282.

[10] D.R.T. Jones, Nucl. Phys. B 87 (1975) 127;

D.R.T. Jones, L. Mezincescu, Phys. Lett. B 138 (1984) 293. 